\title{
Constraints on the equation of state of cold dense matter from nuclear physics and astrophysics
}

\author{
A. F. Fantina ${ }^{1, a}$, N. Chamel ${ }^{1}$, J. M. Pearson ${ }^{2}$, and S. Goriely ${ }^{1}$ \\ ${ }^{1}$ Institut d'Astronomie et d'Astrophysique, CP226, Université Libre de Bruxelles, B-1050 Brussels, Belgium \\ ${ }^{2}$ Département de Physique, Université de Montréal, Montréal (Québec), H3C 3J7 Canada
}

\begin{abstract}
The Brussels-Montreal equations of state of cold dense nuclear matter that have been recently developed are tested against various constraints coming from both nuclear physics and astrophysics. The nuclear physics constraints include the analysis of nuclear flow and kaon production in heavy-ion collision experiments, as well as recent microscopic many-body calculations of infinite homogeneous neutron matter. Astrophysical observations, especially recent neutron-star mass measurements, provide valuable constraints on the high-density part of the equation of state that is not accessible in laboratory experiments.
\end{abstract}

\section{Introduction}

The equation of state $(\mathrm{EoS})$ of dense matter, i.e., the relation between the average baryon number density $n$, the pressure $P$ and the mass-energy density $\rho$, is a crucial ingredient for the description of neutron stars (NSs). These stellar remnants of type II supernova explosions are among the most compact objects in the Universe, with a central density that can reach several times the nuclear matter density (see, e.g., Ref. [1]). The interior of a NS contains at least three distinct regions: (i) the "outer crust", at densities above $\sim 10^{4} \mathrm{~g} \mathrm{~cm}^{-3}$, composed of fully ionised atoms arranged on a regular Coulomb lattice, and neutralised by a degenerate electron gas, (ii) the "inner crust", at densities above $\sim 4 \times 10^{11} \mathrm{~g} \mathrm{~cm}^{-3}$, composed of neutron-proton clusters coexisting with unbound neutrons and electrons, and (iii) the core, at densities above $\sim 10^{14} \mathrm{~g} \mathrm{~cm}^{-3}$, whose composition still remains very uncertain. A consistent treatment of all these different phases is very challenging, but is of utmost importance for studying NS dynamics. For this reason, a family of three different unified EoSs, referred to as BSk19, BSk20, and BSk21, which reflect the current lack of knowledge of high-density matter, has been recently developed [2-4]. These EoSs are tested against different constraints from nuclear physics and astrophysics.

\section{Nuclear physics constraints on the dense-matter equation of state}

The unified Brussels-Montreal EoSs BSk19, BSk20, and BSk21 employed here are based on the nuclear energy-density functional (EDF) theory with generalised Skyrme effective interactions [5],

\footnotetext{
ae-mail: afantina@ulb.ac.be
} 
supplemented with a microscopic contact pairing interaction [6]. These EDFs were fitted to the 2149 measured masses of atomic nuclei with proton number $Z \geq 8$ and neutron number $N \geq 8$ from the 2003 Atomic Mass Evaluation [7], with an rms deviation as low as $0.58 \mathrm{MeV}$. The atomic masses were obtained by adding to the Hartree-Fock-Bogoliubov (HFB) energy a phenomenological Wigner term and a correction term for the collective energy. In addition, the EDFs were constrained to reproduce three different neutron-matter EoSs at $T=0$ : BSk19 (BSk21) was adjusted to an asy-soft (asy-stiff) EoS, while BSk20 was fitted to an EoS with an intermediate stiffness (see Ref. [2] for details).

These neutron-matter EoSs are plotted in Fig. 1, together with recent microscopic many-body calculations employing realistic interactions. The circles and squares correspond respectively to Brueckner-Hartree-Fock (BHF) [8] and Dirac-Brueckner-Hartree-Fock (DBHF) [9] calculations. The shaded area at very low density corresponds to model-independent calculations following the effective field theory approach using the experimental values of the scattering length and effective range [10]. The shaded area extending towards high densities displays the results obtained from auxiliary-field diffusion Monte Carlo calculations by Gandolfi et al. [11]. The calculations performed in Ref. [12] employing the chiral effective field theory are also shown in the same figure. The spread in the results of the microscopic calculations provides an estimate of the current theoretical uncertainties. All of the neutron-matter EoSs obtained with the EDFs BSk19, BSk20, and BSk21 agree quite well with the results obtained in Refs. [10-12]. In particular, the neutron-matter EoS obtained with the BSk21 EDF is in very good agreement with BHF and DBHF EoSs $[8,9]$. This is not surprising since the functional BSk21 has been constrained to reproduce the neutron-matter EoS from Li and Schulze [13] calculated in the BHF approach.

Additional information on the behaviour of high-density matter can be inferred from heavy-ion collision experiments. In particular, constraints on the EoS of symmetric nuclear matter have been obtained from the analysis of nucleon flow data [14] and kaon production [15]. These constraints are represented in Fig. 2 together with the EoSs for symmetric matter obtained with the EDFs BSk19, BSk20, and BSk21. All our three EoSs display a very similar behaviour. The reason for that lies in the fitting procedure adopted in constructing the functionals. Indeed, (i) all three functionals have been fitted to experimental masses [7], including $N \sim Z$ nuclei, (ii) the same value of the symmetry coefficient $J=30 \mathrm{MeV}$ has been imposed, and (iii) the incompressibility of symmetric nuclear matter at saturation was required to fall in the experimental range $240 \pm 10 \mathrm{MeV}$ [16] (see Ref. [2] for details). All three EoSs are compatible with constraints coming from heavy-ion collision experiments in almost all the density range. They are marginally consistent with the EoS extracted from kaon production data around $1.2-1.3 n_{0}$ (being $n_{0}$ the saturation density). However, we emphasise that the constraints of Refs. $[14,15]$ were not directly inferred from experimental data, but were rather determined using specific transport models. Therefore, the uncertainties may have been underestimated.

\section{Astrophysical constraints on the dense-matter equation of state}

With their excellent fit to essentially all experimental atomic masses, and their constraints on the neutron-matter EoSs, the Brussels-Montreal EDFs BSk19, BSk20, and BSk21 are well-suited for a unified description of all regions of a NS. For non-accreting NSs, it is a good approximation to assume that they are made of "cold catalysed matter", i.e. matter in full thermodynamic equilibrium at zero temperature. For the outer crust, the EoS was calculated in the framework of the BPS model [17], using either experimental atomic masses when available or theoretical masses obtained from our HFB mass models (see Ref. [3] for details). The properties of the outer crust in the presence of a strong magnetic field were also studied [18]. For the inner crust, we used the temperature-dependent Thomas-Fermi method extended up to the 4th order, with proton shell corrections added via the Strutinsky integral theorem. Neutron shell effects, which are known to be much smaller, were neglected (see 


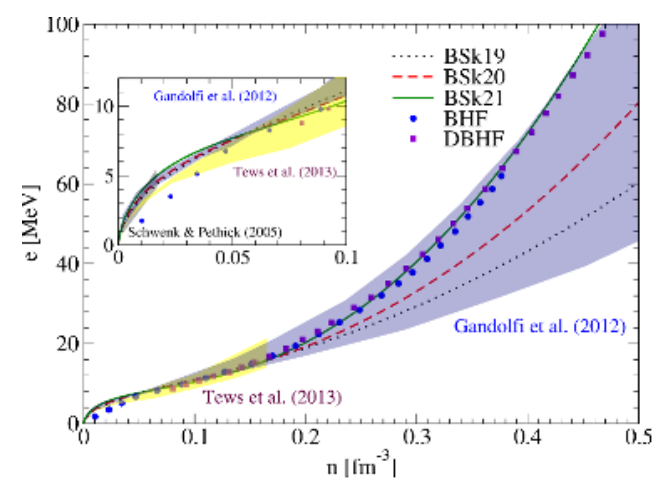

Figure 1. Neutron-matter energy per particle as a function of baryon number density for the EDFs BSk19, BSk20, and BSk21 [2-4]. Circles and squares correspond to BHF [8] and DBHF [9] calculations, respectively. Shaded areas correspond to results obtained from different microscopic manybody calculations [10-12].

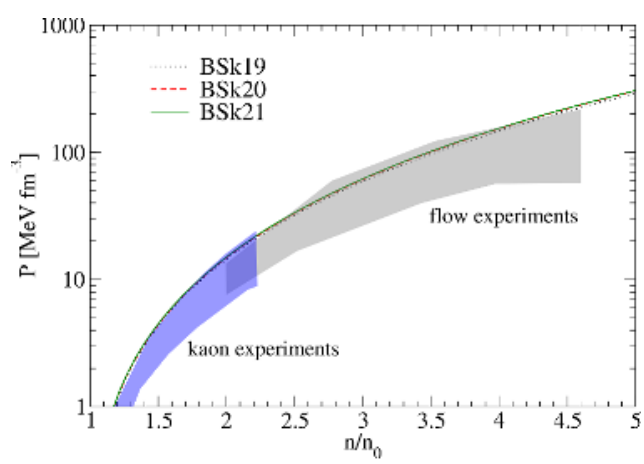

Figure 2. Pressure versus baryon number density (in units of the saturation density $n_{0}$ ) for symmetric nuclear matter for the EDFs BSk19, BSk20, and BSk21 [2-4]. Shaded areas correspond to the constraints obtained from nuclear flow [14] and kaon production [15] in heavy-ion collision experiments.

Ref. [4] for details). This method is a computationally rapid approximation to the full Hartree-Fock equations. In order to further reduce the computations, nuclear clusters were assumed to be spherical, and parametrised nucleon distributions were introduced. Finally, the Wigner-Seitz approximation was used to model the inhomogeneous medium, and pairing effects were neglected. The overall resulting errors in the EoS of the inner crust were found to be about 5\% at the neutron-drip point. The EoS of the core was calculated under the assumption of a uniform plasma of neutrons, protons, electrons and muons in $\beta$ equilibrium [2]. No additional particles were included (see, e.g., Ref. [19] for a discussion of the impact of "exotic" particles on the global NS structure).

Astrophysical observations provide information on the high-density part of the EoS of dense matter, which is not experimentally accessible in the laboratory. Recently, the mass of two NSs in binary systems have been precisely measured: PSR J1614-2230, with a mass $M=1.97 \pm 0.04 M_{\odot}\left(M_{\odot}\right.$ being the mass of our Sun) [20], and PSR J0348+0432, with a mass $M=2.01 \pm 0.04 M_{\odot}$ [21]. These measurements set a lower limit on the maximum NS mass. The question therefore arises whether our EoSs are compatible with these observations. The maximum masses of non-rotating NSs obtained with the Brussels-Montreal EoSs BSk19, BSk20, and BSk21 are respectively $1.86 M_{\odot}, 2.15 M_{\odot}$, and $2.28 M_{\odot}[22]$. Therefore, the asy-softest EoS BSk19 seems to be too soft to support massive NSs with $M \gtrsim 2 M_{\odot}$. Even though the inclusion of rotation increases the maximum mass, the increase amounts to a few percent only [22] for PSR J1748-2446, which is the most rapidly rotating pulsar known with a spin frequency of $716 \mathrm{~Hz}$ [23]. As a consequence, rotation does not change our conclusions. On the other hand, the BSk19 EDF seems to be favoured by the analysis of pion production in heavy-ion collisions, although this analysis still remains a matter of debate. The occurence of a transition to an "exotic" phase in the core of NSs could resolve this apparent discrepancy [19].

\section{Conclusions}

The Brussels-Montreal EoSs of cold dense nuclear matter based on the generalised Skyrme nuclear EDFs, BSk19, BSk20, and BSk21 that were fitted to essentially all experimental atomic mass data, 
are compatible with the most recent microscopic many-body calculations of neutron matter, as well as with empirical constraints on symmetric nuclear matter obtained from the analysis of heavy-ion collisions. On the other hand, recent NS mass measurements indicate that the BSk19 EoS is too soft. This suggests that either the nuclear matter EoS is much stiffer than that obtained with BSk19 or NS cores contain non-nucleonic matter.

\section{Acknowledgements}

The financial support of the F.R.S.-FNRS (Belgium) and the NSERC (Canada) is gratefully acknowledged.

\section{References}

[1] P. Haensel, A. Y. Potekhin, and D. G. Yakovlev, Neutron Stars 1: Equation of State and Structure, Astrophysics and Space Science Library, 326 (Springer, New York, 2007)

[2] S. Goriely, N. Chamel, and J. M. Pearson, Phys. Rev. C 82, 035804 (2010)

[3] J. M. Pearson, S. Goriely, and N. Chamel, Phys. Rev. C 83, 065810 (2011)

[4] J. M. Pearson, N. Chamel, S. Goriely, and C. Ducoin, Phys. Rev. C 85, 065803 (2012)

[5] N. Chamel, S. Goriely, and J. M. Pearson, Phys. Rev. C 80, 065804 (2009)

[6] N. Chamel, Phys. Rev. C 82, 061307 (2010)

[7] G. Audi, A. H. Wapstra, and C. Thibault, Nucl. Phys. A 729, 337 (2003)

[8] W. Zuo, Journal of Physics: Conference Series 420, 012089 (2013)

[9] E. N. E. van Dalen, C. Fuchs, and A. Faessler, Nucl. Phys. A 744, 227 (2004)

[10] A. Schwenk, and C. J. Pethick, Phys. Rev. Lett. 95, 160401 (2005)

[11] S. Gandolfi, J. Carlson, and S. Reddy, Phys. Rev. C 85, 032801 (2012)

[12] I. Tews, T. Krüger, K. Hebeler, and A. Schwenk, Phys. Rev. Lett. 110, 032504 (2013)

[13] Z. H. Li, and H.-J. Schulze, Phys. Rev. C 78, 028801 (2008)

[14] P. Danielewicz, R. Lacey, and W. G. Lynch, Science 298, 1592 (2002)

[15] C. Fuchs, Progress in Particle and Nuclear Physics 56, 1 (2006)

[16] G. Colò, N. van Giai, J. Meyer, K. Bennaceur, and P. Bonche, Phys. Rev. C 70, 024307 (2004)

[17] G. Baym, C. Pethick, and P. Sutherland, Astrophys. J. 170, 299 (1971)

[18] N. Chamel, R. L. Pavlov, L. M. Mihailov, et al., Phys. Rev. C 86, 055804 (2012)

[19] N. Chamel, A. F. Fantina, J. M. Pearson, and S. Goriely, Astron. Astrophys. 553, A22 (2013)

[20] P. B. Demorest, T. Pennucci, S. M. Ransom, M. S. E. Roberts, and J. W. T. Hessels, Nature 467, 1081 (2010)

[21] J. Antoniadis, P. C. C. Freire, N. Wex, et al., Science 340, 1233232 (2013)

[22] N. Chamel, A. F. Fantina, J. M. Pearson, and S. Goriely, Phys. Rev. C 84, 062802(R) (2011)

[23] J. W. T. Hessels, S. M. Ransom, I. H. Stairs, et al., Science 311, 1901 (2006) 